University of Nebraska - Lincoln

DigitalCommons@University of Nebraska - Lincoln

Faculty Publications, Department of Physics and Astronomy

Research Papers in Physics and Astronomy

6-2004

Kapitza-Dirac Diffraction without Standing Waves: Diffraction without a Grating?

\author{
Olga Smirnova \\ Vienna Technical University \\ Daniel L. Freimund \\ University of Nebraska - Lincoln \\ Herman Batelaan \\ University of Nebraska - Lincoln, hbatelaan@unl.edu \\ Misha Ivanov \\ NRC Canada, m.ivanov@imperial.ac.uk
}

Follow this and additional works at: https://digitalcommons.unl.edu/physicsfacpub

Part of the Physics Commons

Smirnova, Olga; Freimund, Daniel L.; Batelaan, Herman; and Ivanov, Misha, "Kapitza-Dirac Diffraction without Standing Waves: Diffraction without a Grating?" (2004). Faculty Publications, Department of Physics and Astronomy. 119.

https://digitalcommons.unl.edu/physicsfacpub/119

This Article is brought to you for free and open access by the Research Papers in Physics and Astronomy at DigitalCommons@University of Nebraska - Lincoln. It has been accepted for inclusion in Faculty Publications, Department of Physics and Astronomy by an authorized administrator of DigitalCommons@University of Nebraska Lincoln. 


\title{
Kapitza-Dirac Diffraction without Standing Waves: Diffraction without a Grating?
}

\author{
Olga Smirnova \\ Photonics Institute, Vienna Technical University, Gusshausstrasse 27/387, A-1040 Vienna, Austria \\ Daniel L. Freimund \\ Department of Physics and Astronomy, University of Nebraska-Lincoln, Lincoln, Nebraska 68588, USA \\ Herman Batelaan \\ Department of Physics and Astronomy, University of Nebraska-Lincoln, Lincoln, Nebraska 68588, USA
}

Misha Ivanov

NRC Canada, 100 Sussex Drive, Ottawa, Ontario K1A 0R6, Canada

(Received 5 December 2003; published 4 June 2004)

\begin{abstract}
We discuss electron diffraction from two counterpropagating light waves with two different frequencies. We show that, even though these waves do not form a standing wave, electron diffraction similar to the conventional Kapitza-Dirac effect, i.e., scattering on a standing wave, is still possible. The nonlinear response of the electron to the laser fields creates a stationary diffraction grating from which the same electron scatters.
\end{abstract}

DOI: 10.1103/PhysRevLett.92.223601

PACS numbers: $33.80 . \mathrm{Rv}, 42.50 . \mathrm{Hz}$

The Kapitza-Dirac effect [1] is the earliest example of the scattering of a matter wave on the periodic spatial structure formed by two counterpropagating light waves, $\mathbf{E}_{0} \cos (\omega t-k x)$ and $\mathbf{E}_{0} \cos (\omega t+k x)$. It is a well-known example where scattering can be described using either the language of photons or the language of diffraction on a stationary ponderomotive potential $U_{p} \propto\left(E_{0} / \omega\right)^{2} \times$ $\cos ^{2}(k x)$ of the standing wave $2 \mathbf{E}_{0} \cos (\omega t) \cos (k x)$. In the photon language, a free electron absorbs a photon from one wave and emits it into the other [Fig. 1(a)], conserving energy and changing momentum by $2 \mathbf{k}$ (atomic units are used throughout). In the diffraction language, the de Broglie electron wave $\left(\lambda_{\mathrm{DB}}=2 \pi / p\right)$ diffracts on the periodic structure formed by the standing wave. The Bragg condition $2 d \sin \theta=\lambda_{\mathrm{DB}}$ for diffraction on a "light crystal" with period $d=\pi / k=\lambda / 2$ coincides with the angle of incidence for which both the energy and the momentum are conserved in the photon picture.

In a general case of two counterpropagating waves with different colors, $\mathbf{E}_{1} \cos \left(\omega_{1} t-k_{1} x\right)$ and $\mathbf{E}_{2} \cos \left(\omega_{2} t+\right.$ $k_{2} x$ ), the energy can be conserved in a multiphoton process - the absorption of $N$ photons $\omega_{1}$ and the emission of $L$ photons $\omega_{2}$-if $N \omega_{1}=L \omega_{2}$. For example, if $\omega_{1}=\omega$ and $\omega_{2}=2 \omega$, absorption of the two $\omega_{1}$ photons and emission of one $\omega_{2}$ photon conserve energy and change the electron momentum by $4 \mathbf{k}$, Fig. 1(b). The angle of incidence for which both the energy and the momentum are conserved is increased by factor two, Fig. 1(b). The photon picture is essentially identical to the conventional Kapitza-Dirac case.

On the other hand, the diffraction picture seems to fail: unless $\omega_{2}=\omega_{1}$, there is no standing wave and there seems to be no stationary diffraction grating associated with it. Is then the conventional Kapitza-Dirac scattering a pathological case, i.e., the only exception where a clear analogy between the photon language and the diffraction language is possible [2]? Are we dealing with stationary scattering without a diffraction grating?

The answer is no. We show that the stationary diffraction grating exists for all cases when $N \omega_{1}=L \omega_{2}$. Furthermore, if the total number of photons involved is odd, as is the case for $\omega_{2}=2 \omega_{1}$ (the absorption of two $\omega_{1}$ photons and the emission of one $\omega_{2}$ photon), the diffraction occurs on a grating, which depends on the initial electron velocity. If, however, the total number of photons involved is even, as is the case for $\omega_{2}=3 \omega_{1}$ (the absorption of three $\omega_{1}$ photons and the emission of one $\omega_{2}$ photon), the grating is velocity independent. Using classical mechanics, we show that the velocity-dependent diffraction in the $\omega_{2}=2 \omega_{1}$ case can be mapped on the

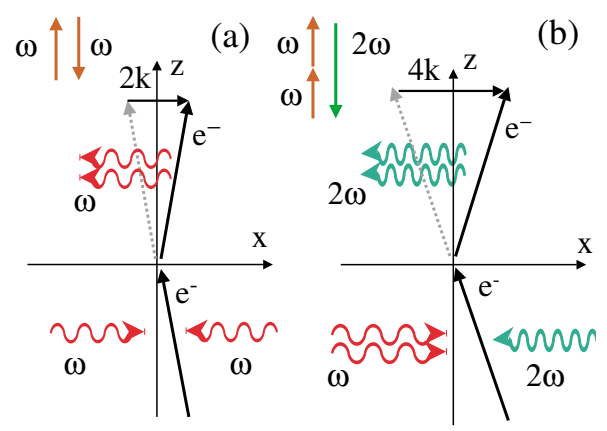

FIG. 1 (color online). (a) Bragg scattering regime for the conventional Kapitza-Dirac effect with one of the two-photon diagrams leading to stationary scattering; (b) Two-color Kapitza-Dirac effect with one of the three-photon diagrams leading to stationary scattering. 
interaction with an effective stationary magnetic field periodic in $x$ (creating velocity-dependent periodic Lorentz force). For an even number of photons, diffraction is mapped onto a velocity-independent stationary potential.

The appearance of the stationary diffraction grating can be understood from a nonlinear optical perspective: the electron both plays the role of a nonlinear medium which forms the grating and also acts as a matter wave (or a classical particle) which diffracts (or deflects) from this grating. Consider, for example, the case of $\omega_{1}=\omega$ and $\omega_{2}=2 \omega$ [Fig. 1(b)]. A linearly polarized field $\omega, k$ propagates from the left along the $x$ axis, while a linearly polarized field $2 \omega, 2 k$ propagates from the right along the same $x$ axis. The electric fields $E_{1}, E_{2}$ are polarized along the $z$ axis, while the magnetic fields $B_{1}, B_{2}$ are polarized along the $y$ axis,

$$
\begin{aligned}
& E(x, t)=E_{1} \cos (\omega t-k x)+E_{2} \cos (2 \omega t+2 k x), \\
& B(x, t)=-B_{1} \cos (\omega t-k x)+B_{2} \cos (2 \omega t+2 k x) .
\end{aligned}
$$

The electron moves in the $x-z$ plane ( $y$ motion remains unaffected).

The electron responds to $E_{1}$ by oscillating along the $z$ axis with frequency $\omega$. The Lorentz force turns it into the figure eight motion along the $z$ axis, where the $2 \omega$ component is along the $x$ axis. Thus, a polarization $P_{x}^{(2)}(2 \omega)=\chi_{x z z}^{(2)}(2 \omega ; \omega, \omega) E_{1, z}^{2}$ is created along the $x$ axis. At the same time, the linear response to $\omega_{2}=2 \omega$ induces polarization $P_{x}^{(1)}(2 \omega)=\chi_{x z}^{(1)}(2 \omega ; 2 \omega) E_{2, z}$ at the same frequency. As in conventional nonlinear optics, mixing the two waves $-P_{x}^{(2)}(2 \omega)$ and $P_{x}^{(1)}(2 \omega)$-induces y polarization grating [3].

Now, similar to conventional wave mixing, the matter wave of the electron diffracts from this stationary grating to generate the new wave; the phase matching is equivalent to the momentum conservation. Conventionally, a nonlinear medium such as a beta barium borate (BBO) crystal is macroscopic, and the momentum recoil cannot be observed. In our case, the nonlinear medium is a single electron, and the momentum recoil is observed as the electron diffraction.

From the nonlinear optics viewpoint, it would perhaps be surprising that the electron can provide the appropriate symmetry for wave mixing to occur [4]. Recall that for $\chi^{2}$ to be nonzero in the dipole approximation the medium cannot be reflection invariant in the $z$ direction. In our case, the nonlinear response arises beyond the dipole approximation and, in general, requires no symmetry breaking. However, the second harmonic component of the figure eight motion is along the $x$ axis. Therefore, the linear response $P_{2}(2 \omega)=\chi_{x z}^{(1)} E_{2}$ is also needed along the $x$ axis, orthogonal to $E_{2}$. The nonzero component $\chi_{x z}^{(1)}(2 \omega ; 2 \omega)$ of the linear susceptibility tensor originates exclusively from the Lorentz force and requires nonzero velocity in the $z$ direction, $\chi_{x z}^{(1)} \propto v_{z}$, breaking the reflection symmetry.

Thus, the strength of the stationary polarization grating and the associated forces acting on the electron will be proportional to its velocity - a situation similar to motion in a stationary magnetic field (see below). This is typical for an odd number of photons.

We first use quantum mechanical perturbation theory to obtain the amplitudes of Kapitza-Dirac-like scattering. We then use classical mechanics to derive effective stationary forces and gratings and show that it is fully consistent with quantum results. The second approach paves the way to a nonperturbative quantum treatment in the exact same way as for the conventional KapitzaDirac effect [5]. Finally, we perform numerical simulations to fully characterize the frequency and intensity dependence of the electron motion.

The Hamiltonian is, in atomic units,

$$
H=\frac{1}{2}\left[\mathbf{p}-\frac{\mathbf{A}_{1}}{c} \sin (\omega t-k x)-\frac{\mathbf{A}_{2}}{c} \sin (2 \omega t+2 k x)\right]^{2},
$$

where $\mathbf{A}_{1}(x, t)$ and $\mathbf{A}_{2}(x, t)$ are the $z$-polarized vector potentials of the electromagnetic fields with amplitudes $A_{1}=c E_{1} / \omega$ and $A_{2}=c E_{2} / 2 \omega$. The Hamiltonian can be written as $H=H_{0}+V$, where $H_{0}=\mathbf{p}^{2} / 2 m$ and $V$ is the rest of the terms in Eq. (2). Among these we find two groups: those oscillating with frequency $\omega\left(V_{\omega}\right)$ and those oscillating with frequency $2 \omega\left(V_{2 \omega}\right)$ :

$$
\begin{aligned}
V_{\omega} & =V_{\omega}^{\prime}+V_{\omega}^{\prime \prime} \\
& =-\frac{\hat{p}_{z} E_{1}}{\omega} \sin (\omega t-k x)-\frac{E_{1} E_{2}}{2 \omega^{2}} \cos (\omega t+3 k x), \\
V_{2 \omega} & =V_{2 \omega}^{\prime}+V_{2 \omega}^{\prime \prime} \\
& =-\frac{\hat{p}_{z} E_{2}}{2 \omega} \sin (2 \omega t+2 k x)-\frac{E_{1}^{2}}{2 \omega^{2}} \cos (2 \omega t-2 k x) .
\end{aligned}
$$

Note the presence of the momentum operator in $V_{\omega}^{\prime}$ and $V_{2 \omega}^{\prime}$. Terms giving nonzero contributions in the lowest order of the time-dependent perturbation theory are proportional to $A_{1}^{2} A_{2}$ and come from the same group ( $V_{\omega}$ or $\left.V_{2 \omega}\right)$. We write the wave function as

$$
\Psi(\mathbf{r}, t)=\frac{1}{L^{3 / 2}} \sum_{p_{n}} c\left(\mathbf{p}_{\mathbf{n}}, t\right) \exp \left[i\left(\mathbf{p}_{\mathbf{n}} \mathbf{r}-\frac{p_{n}^{2}}{2} t\right)\right],
$$

where $L^{3}$ is the quantization volume. Substituting Eq. (4) in the Schrödinger equation with the initial condition $c(\mathbf{p}, 0)=\delta_{\mathbf{p}, \mathbf{p}_{\mathbf{i}}}$, where $\mathbf{p}_{\mathbf{i}}$ is the initial momentum, we obtain for the scattering amplitude $c\left(\mathbf{p}_{f}, t\right)=$ $\delta_{\mathbf{p}_{f}, \mathbf{p}_{\mathbf{i}}}+\delta_{\mathbf{p}_{f}, \mathbf{p}_{i} \pm 4 \mathbf{k}} \Delta c\left(\mathbf{p}_{f}, t\right)+\cdots$. Here $\delta$ is a Kronecker symbol, and the amplitude of scattering $\mathbf{p}_{\mathbf{i}} \rightarrow \mathbf{p}_{\mathbf{i}} \pm 4 \mathbf{k}$ is 


$$
\Delta c\left(\mathbf{p}_{f}\right)=-\sum_{p_{n}} \int d t^{\prime} \int^{t^{\prime}} d t^{\prime \prime} V_{f n}\left(t^{\prime}\right) V_{n i}\left(t^{\prime \prime}\right) e^{i \omega_{f n} t^{\prime}+i \omega_{n i} i^{\prime \prime}},
$$

where $|i\rangle=\left|\mathbf{p}_{i}\right\rangle, \quad|n\rangle=\left|\mathbf{p}_{n}\right\rangle, \quad|f\rangle=\left|\mathbf{p}_{f}\right\rangle=\left|\mathbf{p}_{\mathbf{i}} \pm \mathbf{4 k}\right\rangle$, $\omega_{f n}=p_{f}^{2} / 2-p_{n}^{2} / 2$, and $\omega_{n i}=p_{n}^{2} / 2-p_{i}^{2} / 2$. The stationary contribution appears after the integration over $d t^{\prime \prime}$, if one uses $V^{\prime}$ for one of the matrix elements in conjunction with $V^{\prime \prime}$ from the same group in Eq. (3) for the other matrix element. Momentum conservation appears via the condition that the matrix elements are nonzero.

Finite interaction time due to the laser focus can be included in the impulse approximation. If the initial electron velocity $v_{z}$ is not affected across the focus width $z$, then the amplitudes $E_{1}, E_{2}$ should be replaced by $E_{1} f_{1}\left(t / \tau_{1}\right)$ and $E_{2} f_{2}\left(t / \tau_{2}\right)$, where $f_{l}\left(t / \tau_{l}\right)$ describes focusing of the $l$ th wave with the characteristic interaction time $\tau_{l}=z_{l} / v_{z}$ and $f_{l}(t / \tau)=f_{l}\left(v_{z} t / z_{l}\right)$.

Performing the inner integration, Eq. (5) yields

$$
\Delta c\left(\mathbf{p}_{i} \pm 4 \mathbf{k}\right)= \pm \frac{7 v_{z} E_{1}^{2} E_{2}}{32 \omega^{3} c^{2}} \int_{-\infty}^{\infty} d t f_{1}^{2}(t) f_{2}(t) e^{i \omega_{f i} t},
$$

where $\omega_{f i}=\left(\mathbf{p}_{\mathbf{i}} \pm 4 \mathbf{k}\right)^{2} / 2-p_{i}^{2} / 2$ describes the energy change between the initial and the final states.

As for the traditional Kapitza-Dirac effect, for infinite interaction time $\left(f_{1}=f_{2}=1\right)$ the kinetic energy is conserved [6]: the integral in Eq. (6) yields the delta function $\delta\left(\omega_{f i}\right)$. The condition $\omega_{f i}=0$ can only be satisfied for the Bragg angle [Fig. 1(b)] $\sin \theta_{B}=2 k / p_{i}=2 \lambda_{D B} / \lambda$, analogous to the standard Bragg regime. For sufficiently short interaction time, $\tau$, diffraction is also possible for normal incidence, $\omega_{f i} \tau=8 k^{2} \tau \leq 1$. This is analogous to the standard diffractive regime.

The same result for the scattering amplitude would have been obtained if one were to calculate scattering on the stationary periodic potential

$$
U_{\text {eff }}=\frac{7 v_{z} E_{1}^{2} E_{2}}{16 \omega^{3} c^{2}} \sin 4 k x
$$

using first-order perturbation theory.

We now use classical equations of motion to show explicitly how this potential arises from the nonlinear response of the electron to the laser field. The classical equations of motion are

$$
\ddot{x}=\frac{\dot{z}}{c} B(x, t), \quad \ddot{z}=-E(x, t)-\frac{\dot{x}}{c} B(x, t),
$$

where the $E$ (along $z$ ) and $B$ (along $y$ ) are given by Eq. (1).

To solve these equations we take into account three facts. First, all motions can be separated into fast and slow: $x=x_{f}+x_{s}, z=z_{f}+z_{s}$. Here "fast" refers to oscillations with laser frequencies (and its harmonics), while "slow" refers to the overall motion across the laser focus. Corrections to the slow motion appear only in the third order $\left[\propto E_{1}^{2} E_{2}\right.$; see Eq. (7)]. Therefore, the fast motion can be expanded as $x_{f}=x_{f}^{(1)}+x_{f}^{(2)}+x_{f}^{(3)}+\cdots$ (and the same for $z_{f}$ ). Second, the amplitude of the fast motion is small compared to the wavelength, $k x_{f} \ll 1$ and $k z_{f} \ll 1$, which allows us to expand $E\left(x_{s}+x_{f}, t\right)$ and $B\left(x_{s}+x_{f}, t\right)$ around $x_{s}$. Third, since the velocities of the fast and slow motions are small compared to the speed of light, we can "freeze" the slow coordinate while integrating over one laser cycle, as in the conventional averaging procedure, [7].

We now insert the definition $x=x_{f}+x_{s}, z=z_{f}+z_{s}$ into Eq. (8) and use the Taylor expansion:

$$
\begin{aligned}
& \ddot{x}=\frac{\dot{z}}{c} B\left(x_{f}+x_{s}, t\right) \approx \frac{\dot{z}}{c}\left[B\left(x_{s}, t\right)+x_{f} \frac{\partial B\left(x_{s}, t\right)}{\partial x_{s}}\right] \\
& \ddot{z}=-E\left(x_{s}+x_{f}, t\right)-\frac{\dot{x}}{c} B\left(x_{f}+x_{s}, t\right) \\
& \approx-E\left(x_{s}, t\right)-x_{f} \frac{\partial E\left(x_{s}, t\right)}{\partial x_{s}} \\
& -\frac{\dot{x}}{c}\left[B\left(x_{s}, t\right)+x_{f} \frac{\partial B\left(x_{s}, t\right)}{\partial x_{s}}\right] .
\end{aligned}
$$

Higher order terms in the Taylor expansion yield terms higher in powers of $\dot{x} / c, \dot{z} / c$. Equations (9) and (10) allow one to build perturbative series in powers of the field. It is important to keep terms $\propto \dot{x}_{s} / c, \propto \dot{z}_{s} / c$ on the right-hand side in the first order.

To see how stationary terms arise in the third order in powers of the laser field, let us consider one of them. In the second order, the figure eight motion due to the fundamental field $E_{1},(\omega)$ contains the $2 \omega$ component along the $x$ axis: $\Delta \ddot{x}=-\left(E_{1}^{2} / 2 \omega c\right) \sin (2 \omega t-2 k x)$. In the third order, this $\Delta x_{f}^{(2)}$ is inserted into $\left(\dot{z}_{s} / c\right) x_{f} \partial B\left(x_{s}, t\right) / \partial x_{s}$ on the right-hand side of Eq. (9). In combination with the $2 \omega$ component of $\partial B\left(x_{s}, t\right) / \partial x_{s}$ [equal to $-2 k E_{2} \sin (2 k x+2 \omega t)$ ], it produces stationary term $\propto\left(\dot{z}_{s} / c\right) E_{1}^{2} E_{2} \cos 4 k x$.

Collecting all stationary terms in the third order, we obtain equations for the slow motion:

$$
\begin{gathered}
\ddot{x}_{s}=\left[\frac{q_{x}}{m_{x}}\right] \frac{\dot{z}_{s}}{c} B_{\text {eff }} \cos 4 k x_{s}, \\
\ddot{z}_{s}=-\left[\frac{q_{z}}{m_{z}}\right] \frac{\dot{x}_{s}}{c} B_{\text {eff }} \cos 4 k x_{s},
\end{gathered}
$$

where $B_{\text {eff }}=7 E_{1}^{2} E_{2} /\left(4 \omega^{2} c^{2}\right)$ is the "effective magnetic field," and the "effective charge-to-mass ratios" along $x$ and $z$ are $q_{x} / m_{x}=1$ and $q_{z} / m_{z}=2 / 7$.

These equations look just as if a charged particle was moving in a dc magnetic field directed along the $y$ axis. The only nontrivial aspect is the anisotropy between $x$ and $z$. It arises from $v / c$ anisotropy of the susceptibility tensors (both linear and nonlinear) of the free electron.

We can now identify the effective potential for the slow motion along the $x$ axis. Noting that the right-hand side of 


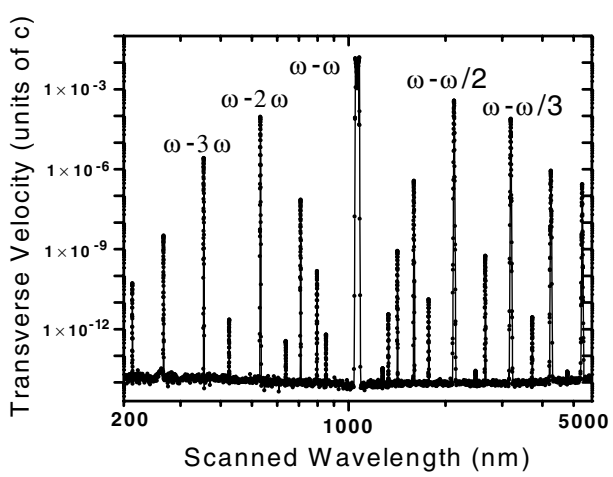

FIG. 2. Wavelength dependence of the electron deflection. One laser wavelength is fixed at $1064 \mathrm{~nm}$; the other is varied. The peaks are labeled on the plot.

Eq. (12) is a full derivative with respect to time and integrating once, we obtain $\dot{z}_{s}=v_{z}-\left(q_{z} / 4 \omega m_{z}\right) B_{\text {eff }} \times$ $\sin 4 k x_{s}$, where $v_{z}$ is the initial velocity along the $z$ axis. Substituting into Eq. (11), we obtain

$$
\ddot{x}_{s}=\frac{v_{z}}{c} \frac{7 E_{1}^{2} E_{2}}{4 \omega^{2} c^{2}} \cos 4 k x_{s}-\frac{7 E_{1}^{4} E_{2}^{2}}{64 c^{5} \omega^{5}} \sin 8 k x_{s} .
$$

For the numerical simulations below, we use typical intensities $I \leq 5 \times 10^{13} \mathrm{~W} / \mathrm{cm}^{2}, v / c \sim 1 / 30$, and $\omega$ corresponding to $1064 \mathrm{~nm}$ light, $\omega \approx 0.043 \mathrm{a}$.u. The first term in Eq. (13) is about 5 orders of magnitude larger than the second term. The effective potential $U_{\text {eff }}(x)=$ $\left(7 v_{z} E_{1}^{2} E_{2}\right) /\left(16 \omega^{3} c^{2}\right) \sin 4 k x$ is the same as identified from the quantum treatment [see Eq. (7)]; it corresponds to the sum of all energy-conserving three-photon diagrams. The second term corresponds to the sum of all secular six-photon diagrams which have zero detuning at the three-photon step.

The intensity and wavelength dependence of the classical motion were checked numerically using Eq. (8). For the wavelength dependence, we fix the wavelength of one laser beam and vary the other, Fig. 2. The initial electron velocity is $10^{7} \mathrm{~m} / \mathrm{s}$, as in the original Kapitza-Dirac experiment [8]. The laser intensity is $5 \times 10^{13} \mathrm{~W} / \mathrm{cm}^{2}$ for both beams, and the interaction time is $3 \mathrm{ps}$. The initial $x$ position of the electron is varied. In Fig. 2 we show the results for that initial $x$ position that gives the maximum deflection in the $x$ direction. When the wavelengths are matched, the usual Kapitza-Dirac effect is present. Strong deflection is also observed whenever $N \omega_{1}=L \omega_{2}$. We checked that the deflection for the $\omega-$ $2 \omega$ peak is in perfect agreement with the prediction based on Eq. (7).

If we direct the initial electron velocity along the $y$ axis so that $v_{z}=0$ but the interaction time is the same, the deflection drops by 5 orders of magnitude for $\omega-2 \omega$ but remains unaffected for $\omega-\omega$ and $\omega-$ $3 \omega$, as expected from Eq. (13). We have also checked

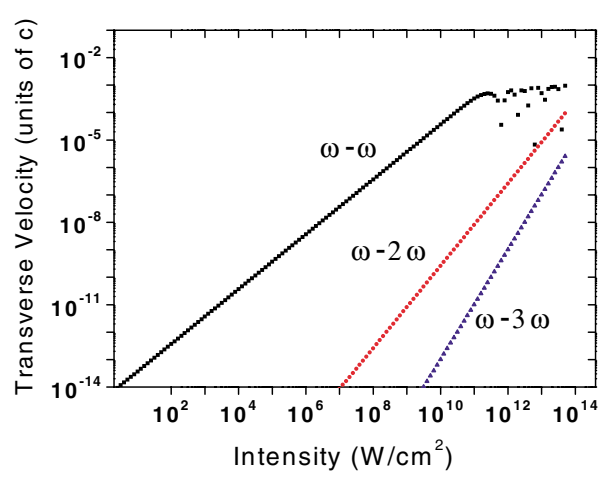

FIG. 3 (color online). Intensity dependence of the electron deflection. The deflection scales with $E^{2}$ for $\omega-\omega$ peak, $E^{3}$ for $\omega-2 \omega$ peak, and $E^{4}$ for $\omega-3 \omega$ peak

that the dependence on the initial velocity, $\boldsymbol{v}_{z}$, is linear for $\omega-2 \omega$. All above parameters are easily accessible experimentally.

Figure 3 shows that the intensity dependence of the deflection at the $\omega-\omega, \omega-2 \omega$, and $\omega-3 \omega$ peaks is $E^{2}, E^{3}$, and $E^{4}$, correspondingly, as expected. At high intensities the deflection is limited for the $\omega-\omega$ peak, due to channeling of the electrons in the valleys of the stationary potential. We have also checked that for $v_{z}=0$ the intensity dependence of the deflection changes to $E^{6}$, in accordance with the second term in Eq. (13).

Exact agreement between the effective potential identified by the classical and quantum treatments allows us to go beyond the perturbative quantum treatment by considering scattering on the effective stationary potential $U_{\text {eff }}$ in Eq. (7) without using perturbation theory, as for the standard Kapitza-Dirac effect [5].

H. B. and D. F. were supported by NSF under Grant No. 0112578 and by DOD-EPSCoR under Grant No. DAAD190210280. M. I. and O. S. were supported by an NSERC IOF grant and O.S. by a Lise Meitner Foundation grant.

[1] P. L. Kapitza and P. A. Dirac, Proc. Cambridge Philos. Soc. 29, 297 (1933).

[2] M.V. Fedorov (private communication).

[3] See, e.g., R. Boyd, Nonlinear Optics (Academic Press, San Diego, CA, 1992).

[4] H. Metcalf and W. Philips (private communication).

[5] M.V. Fedorov, Electrons in a Strong Laser Field (Nauka, Moscow, 1991).

[6] H. Batelaan, Contemp. Phys. 41, 369 (2000).

[7] L. D. Landa and E. M. Lifshitz, Classical Mechanics (Pergamon Press, London, 1960).

[8] D. L. Freimund, K. Aflatooni, and H. Batelaan, Nature (London) 413, 142 (2001). 